\title{
Some Reflections on a Model to Predict the Erosion Rate of the Passive Film on Pure Metals
}

\author{
S. Abdelrahman ${ }^{\dagger}$ and M. M. Stack ${ }^{*}$
}

\begin{abstract}
A model to predict the erosion rates on the passive films of metals is proposed. The model investigates the effect of eroded particles on a metal on which a passive (oxide) film has formed. To date, many of the existing erosion models have concentrated on normal angle erosion of the oxide layer. This new approach considers the effect of oblique impacts on the oxide layer and the effect of its thickness on the amount of metal and oxide layer removal. The model is then tested by comparison to previous experimental work.
\end{abstract}

Keywords: erosion, corrosion, passive layer wear, oblique impacts.

\section{Introduction}

Wastage as a result of the combined effects of the erosion-corrosion by solid particles is a major factor in many industrial situations such as mineral processing and oil and gas exploration. The synergistic effect of both erosion and corrosion may cause rapid metal wastage of components in such environments. In many cases, the surface material forms a passive film during the erosion-corrosion process. However, the removal process involving the transition from erosion of oxide films to the underlying metal is not well understood.

In the literature, Tirupataiah et al. [1] investigated the nature of the elastic rebound on ductile materials when impacted by spherical particles. In this work, an expression was developed for predicting the size of the crater diameter, by equating the kinetic energy of the particle impacting the surface with the energy needed to remove a crater volume from the oxide layer. The model assumed that all the energy of the impact is consumed in the erosion process and the particle was spherical. The basic assumption on which this model is based is that all the relaxation of the crater shape occurs in the direction of the impacting particle.

Later, Roy et. al. [2] investigated the transition effect of the oxide erosion to substrate erosion and developed a similar expression for the crater diameter but for a sharp edged or conical particles and for an oxide layer based on the same first assumption of Tirupataiah. The derivation was applicable for both the substrate (ductile) and the oxide brittle layer. However, the model only considers normal impact and the assumption of the complete consumption of the particle energy in the erosion process ignores any energy expended in elastic work.

\footnotetext{
${ }^{\dagger}$ Research student, University of Strathclyde, s.m.abdelrahman@strath.ac.uk

*Professor, University of Strathclyde, Margaret.stack@strath.ac.uk 
Based on the work of Tirupataiah, previous work involving the current investigators [3] introduced an erosion model for passive layers of metals. The basic assumption was that the rebound velocity is explicitly small compared to the impact velocity and the impact angle is normal. This erosion model was necessary in constructing the erosion-corrosion mapping for various ranges of pure metals and low carbon steel. However, these assumptions constrain the model applicability in dynamic slurry flow motions, as in such environments, the target material is generally exposed to oblique impacts and the rebound characteristics of the impacting particles are considerable. Thus, it is necessary to adapt the model to account for oblique impacts and to account for elastic rebound effects. In addition the model considers the important transition when the eroding particles penetrate an oxide film during the impact process.

\section{Model Assessment}

Starting with the approach of Tirupataiah[1], it is assumed that the energy involved in the erosion process is the difference between the initial impact energy and the energy that causes the particle to rebound namely energy remaining after rebound. It is also assumed that the erosion process is adiabatic, thus discounting the amount of energy consumed during the erosion process due to friction and local melting of the material.

Thus, equating the energy difference by the energy required to form a crater gives:

$$
\frac{m_{p}}{2}\left[V_{1}^{2}-V_{2}^{2}\right]=H U
$$

where $m_{p}$ is the particle mass, $V$ is particle velocity, $H$ is the material hardness, $U$ is the crater volume and the subscripts 1,2 are for the impact and rebound process respectively. Assuming that the surface shear stresses due to oblique impact may be neglected in the case of brittle materials, Finnie[4] stated that the crater diameter of ring crack $d$ is related to the vertical component of the velocity. Then, by default the energy balance in equation (1) will be:

$$
\frac{m_{p}}{2}\left[V_{1}^{2} \sin ^{2} \alpha_{1}-V_{2}^{2} \sin ^{2} \alpha_{2}\right]=H U
$$

where $\alpha_{1}$ and $\alpha_{2}$ are the impact and rebound angles respectively.

By definition, the normal impact velocity component is related to the normal rebound velocity component by the coefficient of restitution $e$, thus and by rearranging:

$$
\frac{m_{p}}{2}\left[V_{1}^{2} \sin ^{2} \alpha_{1}\right]\left(1-e^{2}\right)=H U
$$

Assuming that the particle is a sphere and the crater depth is comparably smaller than the particle diameter, i.e. at low particle velocities, the shape of the crater on the passive film (oxide) surface can be assumed as part of a sphere and $U$ can be related to the crater diameter $W$ by $[1]$ :

$$
U=\frac{\pi W^{4}}{32 d_{p}}
$$


where $d_{p}$ is the particle diameter. Applying equation (4) into (3) gives:

$$
W=1.5023\left[\frac{m_{p} d_{p}\left(1-e^{2}\right)}{H}\right]^{0.25}\left(V_{1} \sin \alpha_{1}\right)^{0.5}
$$

The crater depth $d$ is related to crater diameter using the same assumption above by:

$$
d=\frac{W^{2}}{4 d_{p}}
$$

From Jana [3], the mass of passivating oxide film removed per impact is given as:

$$
M_{t}=\pi k_{2} d_{p} d h \rho_{f}
$$

where $k_{2}$ is constant depending on the substrate material and is given for different pure metals in table. 1 taken from [3], $h$ is the passive layer thickness and $\rho_{f}$ is the passive layer density. Substituting equation (5) into (6) and apply to (7) then we finally get:

$$
M_{t}=1.7726 k_{2} h \rho_{f}\left[\frac{m_{p} d_{p}\left(1-e^{2}\right)}{H}\right]^{0.5}\left(V_{1} \sin \alpha_{1}\right)
$$

Further simplification to the above equation can be made if we put ( $m_{p}=\frac{\pi d_{p}^{3} \rho_{p}}{6}$ ), leads to:

$$
M_{t}=\pi k_{2} h \rho_{f} d_{p}{ }^{2}\left[\frac{\rho_{p}\left(1-e^{2}\right)}{6 H}\right]^{0.5}\left(V_{1} \sin \alpha_{1}\right)
$$

The unit given for the erosion model by equation (9) is $\left[\mathrm{kg} \mathrm{impact}^{-1}\right]$. To convert to a useful unit $\left[\mathrm{kg} \mathrm{m}^{-2} \mathrm{~s}^{-1}\right]$ for example, one should multiply equation (9) by the particle impact frequency. This can be varied according to the erosion-corrosion process being modeled. For example, if the flow is homogeneous (constant particle concentration) then particle impact frequency may be given as [5]:

$$
I_{p}=\frac{c V_{1} \sin \alpha_{1}}{m_{p}}
$$

Table.1: Properties for some pure metals and their passive films taken from [3]

\begin{tabular}{l|l|l|l|l|l|l|l|l|l}
\hline $\begin{array}{c}\text { Metal } \\
\text { Properties }\end{array}$ & $\mathrm{Fe}$ & $\mathrm{Fe}_{2} \mathrm{O}_{3}$ & $\mathrm{Ni}$ & $\mathrm{NiO}$ & $\mathrm{Cu}$ & $\mathrm{Cu} \mathrm{O}^{2}$ & $\mathrm{Al}$ & $\mathrm{Al}_{2} \mathrm{O}_{3}$ & $\begin{array}{c}\text { Silica } \\
\text { particles }\end{array}$ \\
\hline$\rho\left[\mathrm{kg} / \mathrm{m}^{3}\right]$ & 7800 & 5240 & 8900 & 6720 & 8930 & 6400 & 2700 & 3970 & 2670 \\
\hline $\mathrm{k}_{2}$ & 1398.9 & - & 1571.7 & - & 1597.7 & - & 1058.5 & - & - \\
\hline $\mathrm{E}[\mathrm{GPa}]$ & 211 & & 200 & & 130 & & 71 & 380 & 94 \\
\hline $\mathrm{N}$ & 0.293 & & 0.312 & & 0.343 & & 0.345 & 0.22 & 0.3 \\
\hline $\mathrm{H}[\mathrm{MPa}]$ & 820 & 5246.7 & 862 & 6560.9 & 495 & 2736.2 & 260 & 2088.9 & - \\
\hline \hline
\end{tabular}


and $c$ is the particle concentration by mass $\left[\mathrm{kg} \mathrm{m}^{-3}\right]$. The coefficient of restitution (developed elsewhere [6]) is defined as the ratio between the normal component of the particle rebound velocity to that of the impact velocity:

$$
e=\frac{1.36 H^{0.625}}{E_{e}^{0.5} \rho_{p}{ }^{0.125} V_{1}^{0.25}}
$$

Equation (9) is a simple expression for estimating the erosion rates for the oxide or passive layers on a substrate. The equation is valid only for the brittle oxide materials where the deformation even in the oblique impact has an approximate ring shape. The equation is useful for CFD applications since it accounts for the effect of the impact angle in erosion-corrosion evaluation or mapping techniques.

\section{Model Validation and Discussion}

To validate equation (9), experimental work in previous investigations was used to test the validity of the model. Oka et al.[7] investigated the erosion of the aluminum oxide scale at various impact angles. Later, Griffin et al.[8] used his experimental results to validate the results of a three dimensional finite element erosion model of alumina scale on MA956 alloy substrate. Figure 1 shows the experimental results of Oka as reported by Griffin and the prediction by equation (9) after multiplying by $\left[1 /\left(m_{p}{ }^{*} \rho_{f}\right)\right]$ for unit consistency. The experiment involved the impact of quartz particles with $325[\mu \mathrm{m}]$ mean diameter and an impact velocity of $100\left[\mathrm{~m} \mathrm{~s}^{-1}\right]$ on an $\mathrm{Al}_{2} \mathrm{O}_{3}$ scale.

The predicted line here is a function of the scale thickness layer and thus the model agrees well with the experimental results. Although this agreement is sufficient for validating the model, the authors consider more validation through a set of experimental work necessary to investigate the effect of the oxide layer thickness on the model application.

It is possible that very different results may be obtained for erosion of passive films of different density. To date, this has not been considered. In addition, the size of the erosion footprint at various impact angles may have a very significant on the erosion process as it dictates the volume of material removed. Hence, future work will concentrate on the verification of this model through a series of laboratory tests and addressing the above issues.

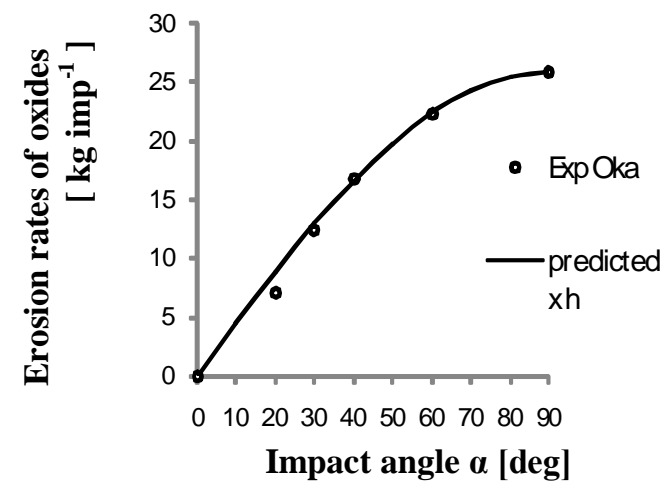

Fig.1: Comparison with the experiment results by Oka et al. [7] 


\section{Conclusions}

(i) A theoretical model has been presented to predict the erosion rates of the passive film which forms on a material instantaneously during exposure to erosioncorrosion environments.

(ii) The model has potential applications to CFD modeling of erosion-corrosion of materials in aqueous slurries.

(iii) Future direction for the research will involve further validation and testing of the model for various metal oxide systems and at different impact angles.

\section{Acknowledgements}

The authors are grateful for the financial support of the Egyptian Government for sponsorship of this $\mathrm{PhD}$.

\section{References}

[1] Tirupataiah, Y., Venkataraman, B. and Sundararajan, G. (1990) The nature of the elastic rebound of a hard ball impacting on ductile metallic gtarget materials. Materials Science and Engineering a-Structural Materials Properties Microstructure and Processing. 124(2) 133-140.

[2] Roy, M., Ray, K. K. and Sundararajan, G. (1998) An analysis of the transition from metal erosion to oxide erosion. Wear. 217(2) 312-320.

[3] Stack, M. M. and Jana, B. D. (2004) Modelling particulate erosion-corrosion in aqueous slurries: some views on the construction of erosion-corrosion maps for a range of pure metals. Wear. 256(9-10) 986-1004.

[4] Finnie, I. (1960) Erosion of surfaces by solid particles. Wear. 3 87-103.

[5] B.T. Lu, J. L. L. (2008) Correlation between repassivation kinetics and corrosion rate over a passive surface in flowing slurry. Electrochimica Acta. 53(23) 7022-7031.

[6] Stack, M. M., Corlett, N. and Zhou, S. (1998) Some thoughts on the effect of elastic rebounds on the boundaries of the aqueous erosion-corrosion map. Wear. 214(2) 175185.

[7] Oka, Y. I., Ohnogi, H., Hosokawa, T. and Matsumura, M. (1997) The impact angle dependence of erosion damage caused by solid particle impact. Wear. 203 573-579.

[8] Griffin, D., Daadbin, A. and Datta, S. (2004) The development of a three-dimensional finite element model for solid particle erosion on an alumina scale/MA956 substrate. Wear. 256(9-10) 900-906. 